Meta

Journal des traducteurs

Translators' Journal

\title{
Accesibilidad en los subtítulos de campañas institucionales contra la violencia de género
}

\section{J. David González-Iglesias González et Antonio J. Martínez Pleguezuelos}

Volume 64, numéro 3, décembre 2019

URI : https://id.erudit.org/iderudit/1070537ar

DOI : https://doi.org/10.7202/1070537ar

Aller au sommaire du numéro

\section{Éditeur(s)}

Les Presses de l’Université de Montréal

ISSN

0026-0452 (imprimé)

1492-1421 (numérique)

Découvrir la revue

Citer cet article

González-Iglesias González, J. D. \& Martínez Pleguezuelos, A. J. (2019).

Accesibilidad en los subtítulos de campañas institucionales contra la violencia de género. Meta, 64(3), 734-747. https://doi.org/10.7202/1070537ar
Résumé de l'article

Dans cet article, nous proposons une analyse du sous-titrage des campagnes institutionnelles menées par l'État espagnol en vue de limiter les problèmes dérivés des violences à caractère sexiste. Concrètement, sur la base des résultats qu'offrent les différentes enquêtes démographiques menées par le gouvernement espagnol et les mesures législatives prises, tant au niveau national qu'européen, nous verrons, dans une perspective intersectionnelle, qu'un important secteur de la population à qui sont adressés les sous-titres sont des femmes qui souffrent de divers degrés de déficience auditive, des femmes qui ne sont pas des locutrices natives de l'espagnol et qui ne possèdent de cette langue que des connaissances limitées, ou une combinaison de ces deux situations. Face à un tel profil, nous examinerons les paramètres appliqués au processus de sous-titrage de ces spots émis en Espagne entre 2006 et 2017 dans l'objectif de vérifier si, selon les caractéristiques techniques établies, l'information est transmise correctement et dans son intégralité au public ciblé ou si elle est au contraire difficile à comprendre pour les secteurs suscités. Pour ce faire, nous étudierons la vitesse de lecture de chaque spot, la présence d'interprète en langue des signes, le narrateur ainsi que d'autres caractéristiques sémiotiques dont on devra tenir compte lors du processus de sous-titrage. 


\title{
Accesibilidad en los subtítulos de campañas institucionales contra la violencia de género*
}

\author{
J. DAVID GONZÁLEZ-IGLESIAS GONZÁLEZ ${ }^{* *}$ \\ Universidad Complutense de Madrid, Madrid, España \\ juandgon@ucm.es
}

\author{
ANTONIO J. MARTÍNEZ PLEGUEZUELOS ${ }^{* *}$ \\ Universidad Complutense de Madrid, Madrid, España \\ antmar17@ucm.es
}

\section{RÉSUMÉ}

Dans cet article, nous proposons une analyse du sous-titrage des campagnes institutionnelles menées par l'État espagnol en vue de limiter les problèmes dérivés des violences à caractère sexiste. Concrètement, sur la base des résultats qu'offrent les différentes enquêtes démographiques menées par le gouvernement espagnol et les mesures législatives prises, tant au niveau national qu'européen, nous verrons, dans une perspective intersectionnelle, qu'un important secteur de la population à qui sont adressés les soustitres sont des femmes qui souffrent de divers degrés de déficience auditive, des femmes qui ne sont pas des locutrices natives de l'espagnol et qui ne possèdent de cette langue que des connaissances limitées, ou une combinaison de ces deux situations. Face à un tel profil, nous examinerons les paramètres appliqués au processus de sous-titrage de ces spots émis en Espagne entre 2006 et 2017 dans l'objectif de vérifier si, selon les caractéristiques techniques établies, l'information est transmise correctement et dans son intégralité au public ciblé ou si elle est au contraire difficile à comprendre pour les secteurs suscités. Pour ce faire, nous étudierons la vitesse de lecture de chaque spot, la présence d'interprète en langue des signes, le narrateur ainsi que d'autres caractéristiques sémiotiques dont on devra tenir compte lors du processus de sous-titrage.

\begin{abstract}
This study presents an analysis of the subtitling of the institutional campaigns promoted by the Government of Spain to fight the problem of gender violence. More specifically, based on the information provided by different demographic surveys carried out by the Spanish Government and by the legislative initiatives issued both at a national and at a European level, we interpret, from an intersectional approach, that a significant part of the population targeted by these subtitles are women with different degrees of hearing disabilities, non-native speakers of Spanish with a limited knowledge of the language, or a combination of both features. In view of this profile, we will analyze the parameters applied in the subtitling process of these advertisements that were aired in Spain between the years 2006 and 2017 in order to verify whether, based on the established technical characteristics, the information is conveyed correctly and completely to the audience, or whether it will be particularly difficult to receive the entire content in the sectors that are included in this study. To do so, we will analyze the reading speed of the subtitles in each advertisement, the presence of a sign language interpreter, the participation of the narrator and other semiotic elements that must be taken into account in the subtitling process.
\end{abstract}

\section{RESUMEN}

En esta investigación proponemos un análisis del subtitulado de las campañas institucionales impulsadas por el Gobierno de España para atajar el problema de la violencia de género. En particular, utilizaremos los datos vertidos por las diferentes encuestas 
demográficas llevadas a cabo por el Gobierno de España y las medidas legislativas impulsadas tanto en el ámbito nacional como en el europeo. Asimismo, entenderemos desde una perspectiva interseccional que un sector importante de la población al que van dirigidos los subtítulos está constituido por mujeres con diferentes grados de discapacidad auditiva y mujeres no nativas de lengua española con un conocimiento limitado de este idioma, así como una combinación de ambos rasgos. Ante este perfil, examinaremos los parámetros aplicados al proceso de subtitulación de estos anuncios emitidos en España desde el año 2006 hasta 2017 con el objetivo de comprobar si, en función de las características técnicas que se han establecido, la información se transmite correctamente y de forma íntegra al público o si resultará complicado recibir todo el contenido para los sectores que tomamos en consideración en este estudio. Para ello, analizaremos la velocidad de lectura de cada anuncio, la presencia de intérprete de signos, el narrador y otros elementos semióticos que deberán tenerse en cuenta en el proceso del subtitulado.

\section{MOTS-CLÉS/KEYWORDS/PALABRAS CLAVE}

accessibilité, sous-titrage, violence de genre, intersectionnalité, campagnes institutionnelles accessibility, subtitling, gender violence, intersectionality, institutional campaigns accesibilidad, subtitulado, violencia de género, interseccionalidad, campañas institucionales

Las personas más vulnerables o que se encuentran expuestas a un riesgo de lesión particularmente elevado, como las sometidas a una violencia reiterada en las relaciones personales, las víctimas de violencia de género o las que son víctimas de otro tipo de delitos [...] deben recibir apoyo especializado y protección jurídica. Los servicios de apoyo especializado deben basarse en un enfoque integrado y preciso que tenga en cuenta, en particular, las necesidades específicas de las víctimas, la gravedad del daño sufrido [...], así como la relación entre las víctimas, los infractores, sus hijos y su entorno social más amplio

(Diario Oficial UE, 2012/29/UE: 61) ${ }^{1}$

\section{Introducción}

Las investigaciones de mediación interlingüística en situaciones de violencia de género que se han desarrollado en el seno de los Estudios de Traducción han profundizado, de manera particular, en enfoques especialmente en el ámbito de la interpretación (Toledano Buendía y Fernández Pérez 2012; Mojica López 2014; Lázaro Gutiérrez y Sánchez Ramos 2015; Lucero García 2016). Sin embargo, existe una gran variedad de discursos audiovisuales que aborda esta problemática con diferentes fines. Desde películas, series o documentales hasta páginas web gubernamentales, encontramos un amplio rango de textos que contribuyen a sensibilizar a la población y a atajar este problema fuertemente arraigado en las sociedades contemporáneas.

Con esta premisa, y a partir de un estudio previo (González-Iglesias y Martínez Pleguezuelos 2016), a continuación, proponemos un análisis de los subtítulos que ofrecen las diferentes campañas contra la violencia de género creadas por el Ministerio de Sanidad, Servicios Sociales e Igualdad del Gobierno de España desde 2006 hasta 2017 con el objetivo de comprobar si su aparición en los diferentes anuncios examinados resulta de utilidad para el público al que van dirigidos. Teniendo en cuenta las necesidades especiales de los usuarios y usuarias de estos subtítulos y la relevancia que puede llegar a tener en un problema como el que tratamos, resulta urgente considerar si la velocidad de lectura de dichos subtítulos, factor que resulta 
clave como veremos a lo largo de la investigación, se ajusta al público meta y si estos cumplen su función adecuadamente con arreglo al resto de parámetros que se analizarán en el estudio.

Cabe pensar que el perfil principal al que van destinados los subtítulos de estas campañas son mujeres con algún tipo de discapacidad auditiva, no nativas de la lengua española o una combinación de ambas por ser el público más vulnerable a este tipo de violencia (Ministerio de Sanidad, Servicios Sociales e Igualdad 2015). Por ello, según las normativas y recomendaciones legales emanadas de instituciones en el nivel estatal y supranacional, estimamos ineludible comprobar si el subtitulado de estos textos audiovisuales traslada a sus usuarios toda la información contenida. No podemos obviar que en muchos casos estos datos ilustran las herramientas sociales y legales que se encuentran a su disposición en los contextos de violencia de género y debemos verificar, por tanto, si los subtítulos son un puente de comunicación oportuno con los usuarios.

\section{Marco jurídico de actuación frente a la violencia de género}

A pesar de los esfuerzos conjuntos que en el nivel estatal y supranacional se están realizando mediante la aprobación e implantación de leyes y directrices para la erradicación de la violencia de género y la protección de sus víctimas, la realidad social en España nos muestra que desde el año 2003 hasta la actualidad 924 mujeres han muerto a manos de sus parejas ${ }^{2}$. Ante estas cifras nefastas, cabe recordar las palabras de Lagarde (1998: 88) cuando señala que los derechos humanos son el resultado de las iniciativas emprendidas para modificar tanto las condiciones genéricas imperantes hoy en día en nuestras sociedades entre hombres y mujeres como, especialmente, las relaciones de poder que las estructuran. En esta dirección, la aprobación de la Ley Orgánica 1/2004, de 28 de diciembre, de Medidas de Protección Integral contra la Violencia de Género facilitó la implantación de un marco jurídico para «prevenir, sancionar y erradicar esta violencia y prestar asistencia a sus víctimas» ${ }^{3}$. Del mismo modo, en Europa, la Directiva 2012/29/UE, de 25 de octubre, del Parlamento Europeo y del Consejo, sustituta de la Decisión marco 2001/220/JAI del Consejo, vio la luz con el objetivo de plantear unas reglas mínimas para defender y proteger los derechos de las víctimas de este tipo de delitos. Este texto no solo «insta a los Estados miembros a que mejoren sus legislaciones y políticas nacionales destinadas a combatir todas las formas de violencia contra la mujer» ${ }^{4}$, sino que, más allá, lista las directrices oportunas para que dicha protección de la mujer sea lo más efectiva posible. De este modo, en su epígrafe 21 se detalla que

[1]a información y las orientaciones brindadas por las autoridades competentes, los servicios de apoyo a las víctimas y de justicia reparadora deben ofrecerse [...] de forma que pueda ser entendida por la víctima, [...] en términos sencillos y en un lenguaje accesible. [...] Debe tenerse en cuenta el conocimiento que tenga la víctima de la lengua utilizada para facilitar información, su edad, madurez, capacidad intelectual y emocional, alfabetización y cualquier incapacidad mental o física. Deben tenerse en cuenta, en particular, las dificultades de comprensión o de comunicación que puedan ser debidas a algún tipo de discapacidad, como las limitaciones auditivas o de expresión oral. ${ }^{5}$

En función de estas indicaciones, entendemos que cualquier acción emprendida con el objetivo de proporcionar auxilio a las víctimas de este tipo de violencia debe 
llegar de manera íntegra a toda la población. Para conseguir estas metas, y a la vista de los nuevos modelos sociales híbridos y fragmentados que configuran la realidad actual de nuestro país, hemos de tener en cuenta aquellos colectivos minoritarios que, por razones de procedencia geográfica, alfabetización o discapacidad física o psíquica, puedan llegar a tener alguna dificultad para recibir el mensaje y, con ello, no emplear oportunamente las herramientas legales y sociales que se encuentran a su alcance. No en vano, basta con una lectura de la macroencuesta del Gobierno español sobre violencia de género para descubrir que «la prevalencia de la violencia de género es invariablemente superior en las mujeres con discapacidad acreditada mayor que el 33\%, sea cual sea el tipo de violencia de género» (Ministerio de Sanidad, Servicios Sociales e Igualdad 2015: 314). También resulta revelador llevar a cabo una revisión de las fichas de víctimas mortales publicadas por el Ministerio de Sanidad, Servicios Sociales e Igualdad para descubrir, por ejemplo, que un 32\% de las 924 mujeres asesinadas en el período cubierto por la estadística estaba constituido por extranjeras. Aunque muchas de ellas tenían un origen hispano, y por tanto contaban con el español como lengua materna, en otros muchos casos las víctimas procedían de países del este de Europa o el norte de África, por lo que el (des)conocimiento de la lengua y la cultura españolas podría erigirse como una barrera a la hora de acceder al mensaje que las campañas de prevención, los textos jurídicos o el propio personal en contacto con estas mujeres pueden ofrecer. De este modo, continuando con las instrucciones que marca la Directiva europea, resulta de suma importancia tener en cuenta a aquellas mujeres que presentan discapacidades físicas o psíquicas, ya sea por problemas relacionados con la vista, por discapacidad mental o por distintos grados de pérdida auditiva, pues son un grupo especialmente vulnerable en los contextos de violencia de género y será necesario encontrar las vías oportunas para transmitir el contenido en las distintas formas de comunicación que se establezcan en el proceso de defensa y protección de la mujer.

Teniendo en cuenta estas premisas, y al amparo de las recomendaciones legales que regulan y promueven la defensa de las mujeres víctimas de violencia de género, partimos en esta investigación del enfoque interseccional feminista que desarrolla Lucero García (2015), aplicado en su estudio a la labor del intérprete en la mediación en casos de violencia de género. En nuestro caso, proponemos ampliar la perspectiva interseccional y trasladarla al campo de la traducción audiovisual con el fin de analizar hasta qué punto el subtitulado en español de las campañas de concienciación en torno a la violencia de género permite a aquellas mujeres hispanohablantes con una lengua materna no española (y que, por tanto, han llegado a ella como segunda lengua y de la que pueden tener un conocimiento básico aunque limitado), a las que presentan una discapacidad auditiva o una combinación de ambos rasgos, recibir todo el contenido informativo que se expone o si, por el contrario, en algunas ocasiones la velocidad de lectura y la ausencia o presencia de otros elementos como la interpretación en lengua de signos impide a estos colectivos seguir el discurso adecuadamente. Para esta tarea, en la que tenemos presentes distintos puntos de vista en función de los diversos perfiles que podemos encontrar entre las mujeres víctimas de estas agresiones, será necesario, por tanto, superar postulados esencializadores en torno a la figura de la mujer que no tengan en cuenta las diferentes aristas que se descubren en un mismo sujeto y que terminan configurando su identidad. 


\section{La mirada interseccional en los contextos de violencia de género}

El concepto de interseccionalidad llegó a los Estudios de Traducción de la mano de autoras como Brufau Alvira $(2009 ; 2010)$ o von Flotow (2009) desde una aproximación de los Estudios de género para entender que la identidad de cada sujeto es la suma de un conjunto heterogéneo e indisoluble de rasgos no excluyentes entre sí. Desde esta perspectiva integral, se tiene en cuenta la confluencia de los distintos ejes que participan en la configuración de un sujeto y que, por ende, condicionan su forma de actuar y relacionarse en sociedad. Rasgos como la raza, la edad, la profesión, el género, el estatus social o la sexualidad contribuyen a perfilar una identidad flexible y voluble, capaz de adaptarse al contexto y a las diferentes circunstancias, haciendo aflorar de forma más visible determinadas características en función de la situación. Para nuestra investigación, consideramos de gran relevancia aplicar este enfoque cuando nos aproximamos al estudio de las vías de comunicación con las víctimas de la violencia de género, ya que existirán diversos condicionantes que tendrán consecuencias tanto en la recepción de los mensajes desde los organismos de prevención y asistencia por parte de las víctimas como en la propia producción (y reescritura) del contenido.

De este modo, más allá de que, como expone la Ley española 1/2004 ya mencionada, la violencia de género se dirija «sobre las mujeres por el hecho mismo de serlo, por ser consideradas, por sus agresores, carentes de los derechos mínimos de libertad, respeto y capacidad de decisión $»^{6}$, según plantea Brufau Alvira (2009: 426) desde el enfoque feminista, las discriminaciones y opresiones que sufren de manera estructural las mujeres se superponen motivadas por las distintas aristas que forman su identidad. Así, el enfoque interseccional considera que una mujer no queda discriminada únicamente por ser mujer, sino que, además, se encuentra expuesta a una doble o triple marginación, en palabras de Tardón Olmos $(2011)^{7}$, en los casos de mujeres inmigrantes que tengan una discapacidad. En los contextos de violencia de género, esta violencia sobre las mujeres es manifiesta a través de los informes que realiza el Ministerio de Sanidad, Servicios Sociales e Igualdad. Así lo refleja la última macroencuesta puesta en marcha por el Gobierno español en 2015, cuando expone, como recogíamos anteriormente, que la violencia de género es superior en mujeres con discapacidad (Ministerio de Sanidad, Servicios Sociales e Igualdad 2015: 314). Tal y como desgrana el informe, la incidencia de la violencia física severa en mujeres discapacitadas dobla a la de las no discapacitadas, con una tasa del $12 \%$ de mujeres con discapacidad que han sufrido violencia física severa, frente al 6,4\% de las mujeres sin discapacidad (Ministerio de Sanidad, Servicios Sociales e Igualdad 2015: 314 y ss.), datos que demuestran que no solo ha sido más frecuente la violencia en este grupo, sino que además con mayor frecuencia ha presentado un carácter más agresivo. En este caso, somos testigos de cómo el carácter interseccional que configura la identidad de estas víctimas contribuye a agravar la situación de estas mujeres, no solo por su condición de mujeres, sino también por su discapacidad.

De forma paralela, la encuesta muestra datos relativos a la violencia sobre mujeres de origen extranjero, con niveles igualmente alarmantes. Si entre las mujeres nacidas en España existe un 11,7\% que ha sufrido maltrato físico, sexual o ambos, la tasa alcanza el 23,5\% para el caso de las nacidas en el exterior (Ministerio de Sanidad, Servicios Sociales e Igualdad 2015: 350 y ss.). En esta situación, el eje cultural que define la identidad de la mujer frente a la del hombre estará muy presente en la con- 
figuración de cada sujeto y el origen social, geográfico y cultural tanto de la víctima como del agresor influirá decisivamente en las relaciones de poder establecidas entre ambos. Ante estas cifras, y considerando los intensos flujos migratorios que arrastra consigo el fenómeno de la globalización y que permite el desplazamiento y la llegada de grandes masas de personas desde puntos geopolíticos y culturales diversos, estimamos acuciante dar un paso al frente y considerar la posición de las mujeres cuya suma de diferentes facetas identitarias las sitúen en una situación más precaria y se vean expuestas en un contexto más complicado si cabe que el resto de la población.

\section{Descripción de los parámetros técnicos aplicados a las campañas contra la violencia de género}

Influidas por las normativas y recomendaciones nacionales e internacionales, y como parte de los compromisos adquiridos por el Estado español, a partir del año 2006 se produjo un cambio significativo en las campañas contra la violencia de género en España, que comenzaron a incluir subtítulos en un esfuerzo por llegar a la población de mujeres sordas y con discapacidad auditiva que pudieran encontrarse en situación de riesgo. Aunque no existen datos específicos acerca del porcentaje de mujeres sordas y con discapacidad auditiva que sufren abusos en el contexto más amplio de las personas con discapacidad, parece razonable suponer que cualquier intento por ofrecerles un mejor acceso a los recursos disponibles solo puede redundar en beneficio de la sociedad en su conjunto. En ese sentido, podemos observar cómo las campañas anuales que se han venido ofreciendo desde el año 2006 -un total de 12 hasta el año 2017- incluyen subtítulos que muestran una transcripción literal de los diálogos que puede escuchar una persona sin discapacidad auditiva.

Los subtítulos literales son una reivindicación tradicional por parte del colectivo de personas sordas (Pereira Rodríguez 2005). Sin embargo, en el contexto de las medidas adoptadas por las instituciones públicas para garantizar la accesibilidad de los contenidos audiovisuales a las personas sordas o con discapacidad auditiva, resulta relevante plantearnos hasta qué punto son útiles los recursos ofrecidos para este público. Para analizar este extremo, debemos tener en cuenta cuáles son los parámetros que determinan si un subtítulo será legible y/o comprensible, sobre todo para la población especialmente vulnerable como la que describíamos con anterioridad, atravesada por características que, de un modo u otro, limitan la velocidad de lectura.

Estudios anteriores al respecto (Díaz Cintas 2005; 2010; Orero 2008; Perego, Del Missier et al. 2010) han determinado una serie de factores que en mayor o menor medida contribuyen a que el texto audiovisual pueda ser asimilado por el público espectador, y en los que se incluyen la selección de la fuente, la longitud de la línea, la duración mínima del subtítulo en pantalla, la pausa mínima entre subtítulos o la velocidad máxima de lectura. En el caso concreto del subtitulado para personas sordas o con discapacidad auditiva, este último factor es probablemente el elemento que condiciona por sí mismo en mayor medida la legibilidad del texto en pantalla. En el campo del subtitulado definimos la velocidad de lectura de un subtítulo como la cantidad de texto que contiene dividido por el tiempo que aparece en pantalla.

En anteriores investigaciones (González-Iglesias 2014) ya mencionábamos, de la mano de Neves, cómo resulta engañoso pensar que el público sordo o con discapacidad auditiva es homogéneo: 
Asumimos que el subtitulado que se ofrece es igualmente adecuado para:

- usuarios sordos y con deficiencia auditiva

- sordos prelocutivos y postlocutivos

- sordos oralistas y signistas

- sordos que se incluyen en la mayoría oyente y Sordos que se incluyen en una minoría lingüística

- sordos para los que el texto escrito es una segunda lengua

- personas con pérdida auditiva con restos auditivos o memoria auditiva

Haciendo esto no estamos ofreciendo nada a nadie (Neves 2008: 26).

De hecho, dentro del campo académico, existen muy diversas opiniones acerca de cuál debería ser la velocidad máxima de lectura en este campo del subtitulado. En una revisión anterior (González-Iglesias y Martínez Pleguezuelos 2016) realizamos un repaso de diferentes voces que se han pronunciado en este sentido, desde aquellas que proponen crear subtítulos diferenciados para los distintos subgrupos contenidos en este sector de la población (sordos postlocutivos y prelocutivos) (Carrera y Lorenzo 2008) hasta la normativa oficial UNE 153010 sobre subtitulado para personas sordas y personas con discapacidad auditiva $(2012)^{8}$, que establece una velocidad de lectura máxima que en ningún caso debería superar los 15 caracteres por segundo (CPS), incluidos espacios en blanco y signos de puntuación. A pesar de que algunos expertos, como Hidalgo Valdés, de la Fundación para la Accesibilidad a los Medios Audiovisuales, propongan una «franja razonable de velocidad entre 17 y 18 [caracteres] por segundo»(2009: 6), que podría llegar incluso a los 20 caracteres por segundo, existen varios estudios de recepción y eye-tracking que revelan que el público, independientemente de que se trate de personas sordas o con discapacidad auditiva o de espectadores oyentes, no es capaz de asimilar de manera consistente los subtítulos con una velocidad de lectura superior a 17-18 CPS (Szarkowska 2016; Miquel Iriarte 2017).

Tras tomar en consideración todos estos parámetros, y en la línea de lo establecido en una revisión anterior (González-Iglesias y Martínez Pleguezuelos 2016), se establecieron en el estudio de análisis de los subtítulos dos valores límite diferentes para la velocidad máxima de lectura de acuerdo con dos grupos hipotéticos diferentes de personas sordas o con discapacidad auditiva, tal y como proponían Carrera y Lorenzo (2008). Un primer valor de 15 CPS se fijó para el grupo de sordos prelocutivos (aquellos que se quedaron sordos antes de adquirir el lenguaje oral), cuyo acceso a la lectoescritura se ha visto necesariamente condicionado por su discapacidad, según se especifica en la norma UNE 153010 (2012) ${ }^{9}$, y para el grupo de sordos postlocutivos (aquellos que se quedaron sordos tras adquirir el lenguaje oral) o sin sordera total se optó por un límite de 17 CPS. Este último valor coincide con el que se emplea normalmente en estudios con un público oyente (Díaz Cintas 2010) y en estudios sobre adecuación de parámetros del subtitulado (González-Iglesias 2014).

Se presenta a continuación un análisis pormenorizado de los diferentes aspectos contemplados en este estudio comparativo: la mención al teléfono de ayuda a las víctimas de malos tratos, la presencia y participación de un intérprete en lengua de signos, la presencia del narrador y la incidencia, evolución e impacto de los subtítulos con una velocidad de lectura superior a lo recomendado para personas sordas o con discapacidad auditiva, así como un apartado específico para el análisis de la última imagen de los anuncios de las diferentes campañas, en los que se concentra la mayor parte de la información de las mismas. 


\section{Presentación de las campañas frente a la violencia de género del Gobierno de España}

\subsection{Teléfono 016}

En cuanto a la información referente al teléfono de atención a víctimas de malos tratos, 016, todas las campañas lo incluyen en su imagen final a partir del año 2007. En ese primer año, el número figura junto al símbolo de un teléfono y, probablemente por tratarse de la primera vez en que este recurso se pone a disposición de las víctimas, la campaña entera gira en torno al servicio telefónico que se ofrece. A partir del año 2008, además del número y del símbolo, se añade el texto "ATENCIÓN VÍCTIMAS VIOLENCIA DE GÉNERO». El texto junto al número se mantiene a lo largo de los años con leves variaciones, y se convierte en "ATENCIÓN A VÍCTIMAS DE MALOS TRATOS POR VIOLENCIA DE GÉNERO» entre 2009 y 2012 y en «ATENCIÓN A VÍCTIMAS DE MALOS TRATOS» entre 2013 y 2017.

Podemos observar, como carencias significativas en cuanto a la ayuda a la accesibilidad de personas sordas o con discapacidad auditiva, que no se informa de la alternativa de la que disponen, por ejemplo, a través del teléfono de texto (DTS), para la que las víctimas deben marcar el número 900116 016. Tampoco se informa en ninguna de las campañas de que el número es gratuito, de que se encuentra disponible las 24 horas del día todos los días del año, ni -quizás el factor más relevante-que este número no queda registrado en la factura telefónica de las líneas fijas y móviles ni en los registros telefónicos de los smartphones.

\subsection{Intérprete de LSE}

En el año 2011 aparece por primera vez una intérprete de lengua de signos española que reproduce el mensaje en la esquina inferior derecha de la pantalla. La presencia de una intérprete no es regular, y se repite en 5 de las 12 campañas (41,67\%). Existen dos aspectos que cabe destacar en esta sección: por un lado, tan solo en una de las 5 campañas se utiliza un intérprete de LSE varón para los anuncios. Por otra parte, en las campañas con subtítulos traducidos a otras lenguas del Estado español, así como al inglés o al francés, se mantiene intacta la imagen del o de la intérprete, de manera que la lengua de signos que se ofrece es en todos los casos la española, con lo que no se transmite dicha información a través de lengua de signos catalana (LSC), valenciana (LSCV), ni a cualquiera de las lenguas de signos de países de habla inglesa o francesa.

\subsection{Narrador}

En el conjunto de anuncios estudiados a lo largo de las diferentes iniciativas se observa un cambio notable a partir del año 2011 en lo relativo a la voz que narra el mensaje central de cada una de las campañas. Desde su comienzo en el año 2006, distintas voces masculinas estuvieron a cargo de esta tarea, y en el año 2011 se pudo escuchar por primera vez a una mujer como la voz en off que informaba a la audiencia acerca del eslogan contra la violencia de género elegido. Quizás este cambio no sea casual, ya que tal y como afirma Lázaro Gutiérrez $(2018)^{10}$, en muchas ocasiones en las que era necesaria la participación de un intérprete en tribunales u hospitales 
en contextos de violencia de género, la mujer víctima de las agresiones no se sentía cómoda con un intérprete varón. Por ello, en algunos casos se detectaba que la víctima se encontraba cohibida y no relataba los episodios de violencia con la intensidad necesaria, o directamente solicitaban una intérprete mujer. Por último, cabe destacar la excepción que supone la campaña del año 2017, que se basa íntegramente en la presentación de texto en pantalla y que no incluye voces de ningún tipo.

\subsection{Subtítulos rápidos}

Tras la determinación de un valor máximo como umbral para el conjunto de sordos prelocutivos y de otro valor máximo, mayor en este caso, para el grupo de sordos postlocutivos o personas con discapacidad auditiva, se hacía necesario obtener los subtítulos en un formato que permitiera su análisis cuantitativo de forma sistematizada. En el caso que nos ocupa, el texto de los anuncios se incluyó como subtítulos abiertos, que son aquellos que forman parte indisoluble de la imagen y que no pueden separarse de ella como un archivo independiente. Por este motivo, fue necesario duplicar manualmente el texto mediante software especializado, de tal modo que el archivo resultante fuese una réplica exacta de los subtítulos que aparecían en pantalla.

A continuación, sometimos a los subtítulos a un análisis a través de un programa específicamente diseñado para ello (González-Iglesias 2015) y con el que se puede obtener, de manera sistematizada, la información numérica correspondiente a diferentes parámetros técnicos, entre los que se encuentra la velocidad de lectura. Además de conocer este último dato, el software nos permite extraer el porcentaje de subtítulos que superan un valor límite dado, que en el caso de nuestro estudio fue de 17 CPS para los subtítulos rápidos para un público de sordos postlocutivos (SR-Pos) y de 15 CPS para los subtítulos rápidos para un público de sordos prelocutivos (SR-Pre).

Como resultado del análisis, pudimos observar un período con una tendencia clara a incluir un porcentaje cada vez mayor de subtítulos con velocidades de lectura superiores a las fijadas, entre 2006 y 2013, seguido por un segundo tramo en el que estos porcentajes se ven reducidos sensiblemente, entre 2013 y 2017. El Cuadro 1 presenta una visión general de las diferentes características incluidas en el análisis, con dos categorías diferenciadas para el porcentaje de subtítulos rápidos para sordos postlocutivos y para sordos prelocutivos. Los valores numéricos hacen referencia a la media de cada una de las campañas anuales.

\section{Cuadro 1}

Análisis de las variables de los anuncios en las diferentes campañas contra la violencia de género

\begin{tabular}{|l|c|c|l|c|c|}
\hline Año & Información 016 & Intérprete LSE & Narrador & \%SR-Pos & $\%$ SR-Pre \\
\hline 2006 & No & No & Hombre & 37,03 & 81,48 \\
\hline 2007 & Sí & No & Hombre & 50,00 & 60,00 \\
\hline 2008 & Sí & No & Mixto & 31,56 & 71,21 \\
\hline 2009 & Sí & No & Hombre & 75,00 & 85,71 \\
\hline 2010 & Sí & No & Hombre & 54,39 & 66,95 \\
\hline 2011 & Sí & Mujer & Mujer & 58,03 & 71,57 \\
\hline 2012 & Sí & Mujer & Mujer & 58,82 & 82,35 \\
\hline 2013 & Sí & No & Mujer & 77,78 & 100,00 \\
\hline
\end{tabular}




\begin{tabular}{|l|l|l|l|l|l|}
\hline 2014 & Sí & Mujer & Mujer & 72,69 & 92,59 \\
\hline 2015 & Sí & Mujer & Mujer & 46,41 & 55,51 \\
\hline 2016 & Sí & No & Mujer & 46,64 & 50,48 \\
\hline 2017 & Sí & Hombre & No hay & 12,50 & 25,00 \\
\hline
\end{tabular}

\%SR-Pos: Porcentaje de subtítulos rápidos para sordos postlocutivos, por encima de 17 CPS; \%SR-Pre: Porcentaje de subtítulos rápidos para prelocutivos, por encima de 15 CPS

Así, en el caso de los subtítulos rápidos para sordos postlocutivos se comienza con campañas en las que entre un tercio y la mitad de los subtítulos cuentan con una velocidad de lectura por encima de los 17 CPS, que pasan a representar una proporción aún mayor en años siguientes, para después rebajarse de forma gradual hasta la última campaña analizada, en la que tan solo representan un $12,50 \%$.

Para los sordos prelocutivos, la situación es todavía más crítica. Las proporciones de subtítulos que son demasiado rápidos para permitir la comprensión de lo que se pretende transmitir siguen una evolución similar, con un crecimiento constante entre 2006 y 2012 hasta llegar al 100\% de subtítulos por encima de los 15 CPS en 2013. A partir de ese momento, las cifras bajan considerablemente hasta un 25,00\% en el año 2017.

El caso de la última campaña, correspondiente al año 2017, y que presenta los valores más bajos de subtítulos rápidos para cualquiera de las dos poblaciones consideradas en el período de nuestro estudio, es excepcional en cuanto a sus características. Se trata de un único anuncio de 20 segundos sin diálogos hablados ni locución de ningún tipo en el que se suceden las imágenes de personas del entorno próximo de un maltratador junto con una leyenda que indica la naturaleza de su relación con él (padre, vecina, compañero), seguidas del mensaje central de la campaña: «Cuando hay maltrato en una pareja, no son solo cosas de pareja. No permitas la violencia de género». Como resultado del formato de esta campaña, nos encontramos con un anuncio en el que la velocidad de lectura del texto que aparece en pantalla, que por primera vez no es la transcripción de una voz que los espectadores oyentes puedan escuchar, se ajusta mucho más que en años anteriores a los parámetros de aceptabilidad que hemos definido anteriormente.

\subsection{Imagen final}

Mención aparte merece la imagen final correspondiente a cada uno de los anuncios de las diferentes campañas, por tratarse del momento en el que confluye la mayor cantidad de información durante un lapso de tiempo muy breve. En este punto, la pantalla presenta de manera simultánea tres focos de información gráfica: por una parte, y por lo general con un tipo de letra de mayor tamaño y en la parte central de la imagen, encontramos el mensaje central de la campaña correspondiente a ese año. Por otro lado, situado en el margen inferior o derecho de la pantalla, y ya con una letra de mucho menor tamaño, podemos ver la imagen institucional del Gobierno de España con mención al ministerio específico responsable de la campaña (recordemos que las campañas contra la violencia de género han sido emitidas desde una oficina que a lo largo de los últimos 12 años ha pertenecido a cuatro denominaciones ministeriales diferentes), y por último, junto a esta, la imagen de información acerca del número 016, en el que como ya hemos indicado anteriormente, se añade un breve texto que indica la atención a víctimas de malos tratos por violencia de género. A 
estos tres elementos hay que añadir un cuarto: los subtítulos que siguen acompañando la voz en off, y que en muchos casos ofrecen una información redundante que aparece ya en pantalla.

En este sentido, a pesar de que durante todos los anuncios existe una coincidencia plena entre lo que se lee en la imagen y lo que narra la voz, encontramos en este punto el único momento de las campañas en las que se aprecia un desajuste: aunque los narradores mencionan el eslogan de las campañas y acaban los anuncios con la indicación «Gobierno de España», no señalan la existencia del teléfono 016, cuya información solo aparece por escrito.

Como resultado de todo ello, nos encontramos en la imagen final con una saturación de elementos textuales y gráficos que se muestran durante un lapso de tiempo que en ningún caso excede 1,5 segundos. En nuestro análisis, hemos dedicado un apartado propio al estudio de la velocidad de lectura de esta última imagen en las diferentes campañas. El Cuadro 2 muestra la velocidad de lectura, medida en caracteres por segundo, de la última imagen de cada uno de los anuncios. Los valores numéricos hacen referencia a la media de cada una de las campañas anuales.

\section{CUADRO 2}

Media anual de la velocidad de lectura de la última imagen de las diferentes campañas

\begin{tabular}{|l|c|}
\hline Año & Velocidad de lectura última imagen \\
\hline 2006 & 28,94 \\
\hline 2007 & 49,03 \\
\hline 2008 & 35,27 \\
\hline 2009 & 72,94 \\
\hline 2010 & 61,14 \\
\hline 2011 & 46,14 \\
\hline 2012 & 48,28 \\
\hline 2013 & 107,87 \\
\hline 2014 & 59,44 \\
\hline 2015 & 94,18 \\
\hline 2016 & 114,61 \\
\hline 2017 & 50,33 \\
\hline
\end{tabular}

Como podemos apreciar en el cuadro, incluso en el mejor de los casos (correspondiente a la campaña del año 2006, en la que no se incluía información acerca del teléfono 016), la velocidad de lectura supera los 28 caracteres por segundo, más del doble de lo que una persona con sordera prelocutiva sería capaz de interpretar, y desde luego por encima de lo que incluso una persona oyente o con sordera postlocutiva puede asimilar. Estos valores aumentan en algunos casos hasta rozar niveles que superan 10 veces los máximos recomendados.

Podemos, pues, concluir que para un espectador cualquiera, y muy especialmente para uno con sordera o discapacidad auditiva, la saturación de información provocada por la combinación de subtítulos y texto en pantalla hace que resulte de todo punto imposible comprender el mensaje que se pretende transmitir. Cabría preguntarse si no es precisamente este momento de la campaña, en el que se concentran el eslogan y la información de interés para el espectador, el que debería ofrecer una velocidad de lectura más accesible para todos. 


\section{Conclusiones}

A partir de todo lo anterior, constatamos en la implementación de estas políticas de accesibilidad que, si bien la intención es por supuesto loable, se ve lastrada por consideraciones ineludibles de carácter técnico que anulan en cierta medida el efecto que se pretende conseguir. Nos encontramos con un caso muy ilustrativo del principio fundamental de que la práctica debe ir de la mano de la teoría si queremos un resultado eficaz. De no ser así, corremos el riesgo de limitar el acceso a la información a aquellos sectores de la población más vulnerables.

En concreto, en el estudio que hemos desarrollado y como describíamos al comienzo de este trabajo, la posición de las víctimas es especialmente delicada en los casos de mujeres con discapacidades auditivas, no nativas de lengua española o con ambas características. Tal y como muestran los datos alcanzados en el estudio desarrollado, consideramos urgente prestar atención a las necesidades de estos sectores específicos para brindarles la misma información y conseguir que tanto el mensaje de concienciación como el contenido sobre las herramientas legales lleguen de manera adecuada a todos los destinatarios, especialmente a aquellas mujeres víctimas de la violencia de género. Si no prestamos atención a las dificultades que plantea el seguimiento de subtítulos demasiado rápidos para la población sorda, se corre el riesgo de estar proyectando un contenido que no llega a resultar útil para aquellos a los que va destinado.

\section{AGRADECIMIENTOS}

El presente artículo se inscribe en el proyecto de investigación «Violencia simbólica y traducción: retos en la representación de identidades fragmentadas en la sociedad global» (FFI2015-66516-P; MINECO / FEDER, UE), financiado por el Ministerio de Economía y Competitividad del Gobierno de España y el Fondo Europeo de Desarrollo Regional.

\section{NOTAS}

* El trabajo de investigación, objeto del presente artículo, ha sido efectuado mientras Juan David González-Iglesias González y Antonio Jesús Martínez Pleguezuelos estaban afiliados a la Universidad Autónoma de Madrid y a la Universidad de Alcalá de Henares, respectivamente.

** Grupo de Investigación Reconocido TRADIC «Traducción, Ideología y Cultura» (Universidad de Salamanca).

1. Diario Oficial de la Unión Europea, Directiva 2012/29/UE del Parlamento Europeo y del Consejo, de 25 de octubre de 2012, por la que se establecen normas mínimas sobre los derechos, el apoyo y la protección de las víctimas de delitos. Diario Oficial de la Unión Europea, L 315, del 14 de noviembre de 2012: 57-73.

2. Información recogida en la página web del Ministerio de Sanidad, Servicios Sociales e Igualdad del Gobierno de España en las fichas estadísticas de víctimas mortales por violencia de género desde el año 2003 (http://www.violenciagenero.msssi.gob.es/violenciaEnCifras/victimasMortales/ fichaMujeres/home.htm). Datos consultados a 27 de abril de 2018.

3. Boletín Oficial del Estado. Ley Orgánica 1/2004, de 28 de diciembre, de Medidas de Protección Integral contra la Violencia de Género. Boletín Oficial del Estado, núm. 313, de 29 de diciembre de 2004: 42166-42197.

4. Diario Oficial de la Unión Europea, Directiva 2012/29/UE del Parlamento Europeo y del Consejo, de 25 de octubre de 2012, por la que se establecen normas mínimas sobre los derechos, el apoyo y la protección de las víctimas de delitos. Diario Oficial de la Unión Europea, L 315, del 14 de noviembre de 2012: 57-73.

5. Diario Oficial de la Unión Europea, Directiva 2012/29/UE del Parlamento Europeo y del Consejo, de 25 de octubre de 2012, por la que se establecen normas mínimas sobre los derechos, el apoyo y 
la protección de las víctimas de delitos. Diario Oficial de la Unión Europea, L 315, del 14 de noviembre de 2012: 57-73.

6. Boletín Oficial del Estado. Ley Orgánica 1/2004, de 28 de diciembre, de Medidas de Protección Integral contra la Violencia de Género. Boletín Oficial del Estado, núm. 313, de 29 de diciembre de 2004: 42166-42197.

7. Tardón Olmos, María (2011): Mujeres con discapacidad y violencia de género, no publicada. IV Congreso del Observatorio contra la violencia de doméstica y de género. Madrid, 16-17 de noviembre de 2011.

8. AENOR (2012): Norma Española UNE 153010:2012. Subtitulado para personas sordas y personas con discapacidad auditiva. Madrid: Asociación Española de Normalización y Certificación.

9. AENOR (2012): Norma Española UNE 153010:2012. Subtitulado para personas sordas y personas con discapacidad auditiva. Madrid: Asociación Española de Normalización y Certificación.

10. Lázaro Gutiérrez, Raquel (2018): Interpretación en los Servicios Públicos, no publicada. Mujer y Traducción. Interpretación en casos de violencia de género. Alcalá de Henares, 14 de marzo de 2018.

\section{REFERENCIAS BIBLIOGRÁFICAS}

Brufau Alvira, Nuria (2009): Traducción y género: propuestas para nuevas éticas de la traducción en la era del feminismo transnacional. Tesis de doctorado. Salamanca: Universidad de Salamanca.

Brufau Alvira, Nuria (2010): Interviewing Luise von Flotow. A New State of the Art. Quaderns. Revista de traducció. 17:283-292.

CARrera, Judith y Lorenzo, Lourdes (2008): Variedades de español en subtitulación para sordos. In: Catalina Jiménez Hurtado y Ana Rodríguez Domínguez, ed. Accesibilidad a los contenidos audiovisuales para personas con discapacidad Amadis '07. Madrid: Real Patronato sobre Discapacidad, 79-88.

Díaz Cintas, Jorge (2005): The Ever-changing World of Subtitling: Some Major Developments. In: John D. SANDERSON, ed. Research on Translation for Subtitling in Spain and Italy. Alicante: Publicaciones de la Universidad de Alicante, 17-26.

Díaz Cintas, Jorge (2010): Subtitling. In: Yves Gambier y Luc van Doorslaer, ed. Handbook of Translation Studies. Manchester: St Jerome, 344-349.

GonzÁlez-Iglesias, J. David (2014): Desarrollo de una herramienta de análisis de los parámetros técnicos de los subtítulos y estudio diacrónico de series estadounidenses de televisión en DVD. Salamanca: Ediciones Universidad de Salamanca.

GonzÁlez-Iglesias, J. David (2015): Software Tools for the Analysis of Technical Parameters of Subtitles. In: Anna Jankowska y Agnieszka Szarkowska, ed. New Points of View on Audiovisual Translation and Media Accessibility. Berna: Peter Lang, 47-56.

González-Iglesias, J. David y Martínez Pleguezuelos, Antonio J. (2010): Gender Violence Campaigns and Accessibility to Information in the Media. In: Martin Kažımír, ed. Kontexty. Interdisciplinárny zborník. Gorlice: Elpis, 237-249.

Hidalgo Valdés, Miguel (2009): Estudio sobre la velocidad de los subtítulos en televisión para personas sordas. Sevilla: Fundación para la Accesibilidad a los Medios Audiovisuales.

LAGARDE, Marcela (1998): Identidad de género y derechos humanos. In: Laura GuzMán STEIN y Gilda Pacheco Oreamuno, ed. Estudios Básicos de Derechos Humanos IV. Instituto Interamericano de Derechos Humanos / Comisión de la Unión Europea: San José de Costa Rica, 85-125.

LÁzaro Gutiérrez, Raquel y Sánchez Ramos, María del Mar (2015): Corpus-Based Interpreting Studies and Public Service Interpreting and Translation Training Programs: The Case of Interpreters Working in Gender Violence Contexts. In: Jesús Romero-Trillo, ed. Yearbook of Corpus Linguistics and Pragmatics 2015. Current Approaches to Discourse and Translation Studies. Dordrecht: Springer, 275-292.

Lucero García, Marta (2015): Interseccionalidad y Feminist-Relational Approach: nuevos enfoques para la formación y actuación de intérpretes en contextos de violencia de género. Asparkía. 26:91-104. 
Lucero García, Marta (2016): Cuestiones de identidad. Estudio teórico sobre el papel del intérprete judicial en contextos de violencia de género. Parallèles. 28(2):91-104.

Ministerio de Sanidad, Servicios Sociales e Igualdad (2015): Macroencuesta de violencia contra la mujer 2015. Madrid: Ministerio de Sanidad, Servicios Sociales e Igualdad. Centro de publicaciones.

Miquel Iriarte, Marta (2017): The Reception of Subtitling for the Deaf and the Hard of Hearing: Viewers' Hearing and Communication Profile \& Subtitling Speed of Exposure. Tesis de doctorado. Barcelona: Universitat Autònoma de Barcelona.

Mojica López, Esperanza (2014): Análisis de la situación de la traducción y la interpretación en el ámbito judicial en España en casos específicos de violencia de género. FITISPos International Journal, 1(1):169-180.

Neves, Josélia (2008): Inexactitudes sobre el subtitulado para sordos y discapacitados auditivos. In: Catalina Jiménez Hurtado y Ana Rodríguez Domínguez, ed. Accesibilidad a los contenidos audiovisuales para personas con discapacidad Amadis '07. Madrid: Real Patronato sobre Discapacidad, 23-35.

Orero, Pilar (2008): Le format des sous-titres: les mille et une possibilités. In: Jean Marc LAVAUR y Adriana ŞERBAn, ed. La traduction audiovisuelle: Approche interdisciplinaire du soustitrage. Bruselas: De Boeck Université, 55-67.

Perego, Elisa, Del Missier, Fabio, Porta, Marco, et al. (2010): The Cognitive Effectiveness of Subtitle Processing. Media Psychology. 13(3):243-272.

Pereira Rodríguez, Ana (2005): El subtitulado para sordos: estado de la cuestión en España. Quaderns. Revista de traducció. 12:161-172.

SzarkowsKa, Agnieszka (2016): Report on the Results of an Online Survey on Subtitle Presentation Times and Line Breaks in Interlingual Subtitling. Part 1: Subtitlers. London: Centre for Translation Studies.

Toledano Buendía, Carmen y Fernández Pérez, Marlene (2012): Las barreras lingüísticas en la atención a las víctimas extranjeras de violencia de género: un factor ignorado. In: María José Tacoronte Domínguez, Anisa Azaovagh de la Rosa y Amparo Romero Sánchez, ed. Actas II Jornadas de Investigaciones Feministas y Análisis de Género. Avances y propuestas. II Jornadas de Investigaciones Feministas y Análisis de Género. Avances y Propuestas, San Cristóbal de La Laguna, 8-9 de octubre de 2012. La Laguna: Instituto Universitario de Estudios de las Mujeres, 33-44.

von Flotow, Luise (2009): Contested Gender in Translation: Intersectionality and Metramorphics. Palimpsestes. 22:245-256. 